\title{
Basset: learning the regulatory code of the accessible genome with deep convolutional neural networks
}

\author{
David R. Kelley, ${ }_{1}^{1}$ Jasper Snoek, ${ }^{2}$ and John L. Rinn ${ }^{1}$ \\ ${ }^{1}$ Department of Stem Cell and Regenerative Biology, Harvard University, Cambridge, Massachusetts 02138, USA; \\ ${ }^{2}$ School of Engineering and Applied Science, Harvard University, Cambridge, Massachusetts 02138 , USA
}

\begin{abstract}
The complex language of eukaryotic gene expression remains incompletely understood. Despite the importance suggested by many noncoding variants statistically associated with human disease, nearly all such variants have unknown mechanisms. Here, we address this challenge using an approach based on a recent machine learning advance-deep convolutional neural networks (CNNs). We introduce the open source package Basset to apply CNNs to learn the functional activity of DNA sequences from genomics data. We trained Basset on a compendium of accessible genomic sites mapped in 164 cell types by DNase-seq, and demonstrate greater predictive accuracy than previous methods. Basset predictions for the change in accessibility between variant alleles were far greater for Genome-wide association study (GWAS) SNPs that are likely to be causal relative to nearby SNPs in linkage disequilibrium with them. With Basset, a researcher can perform a single sequencing assay in their cell type of interest and simultaneously learn that cell's chromatin accessibility code and annotate every mutation in the genome with its influence on present accessibility and latent potential for accessibility. Thus, Basset offers a powerful computational approach to annotate and interpret the noncoding genome.
\end{abstract}

[Supplemental material is available for this article.]

The process of identifying genomic sites that show statistical relationships to phenotypes holds great promise for human health and disease (Hindorff et al. 2009). However, our current inability to efficiently interpret noncoding variants impedes progress toward using personal genomes in medicine. Coordinated efforts to survey the noncoding genome have shown that sequences marked by DNA accessibility and certain histone modifications are enriched for variants that are statistically related to phenotypes (The ENCODE Project Consortium 2012; Roadmap Epigenomics Consortium et al. 2015). The first stages of a mechanistic hypothesis can now be assigned to variants that directly overlap these annotations (Fu et al. 2014; Kircher et al. 2014; Ritchie et al. 2014).

However, simply considering the overlap of a variant with annotations underutilizes these data; more can be extracted by understanding the DNA-protein interactions as a function of the underlying sequence. Proteins that recognize specific signals in the DNA influence its accessibility and histone modifications (Voss and Hager 2014). Given training data, models parameterized by machine learning can effectively predict protein binding, DNA accessibility, histone modifications, and DNA methylation from the sequence (Das et al. 2006; Arnold et al. 2013; Benveniste et al. 2014; Pinello et al. 2014; Lee et al. 2015; Setty and Leslie 2015; Whitaker et al. 2015). A trained model can then annotate the influence of every nucleotide (and variant) on these regulatory attributes. This upgrades previous approaches in two ways. First, variants can be studied at a finer resolution; researchers can prioritize variants predicted to drive the regulatory activity and devalue those predicted to be irrelevant bystanders. Second, rare variants that introduce a gain of function will often not overlap regulatory annotations in publicly available data. An accurate model for

\section{Corresponding author: dkelley@fas.harvard.edu}

Article published online before print. Article, supplemental material, and publication date are at http://www.genome.org/cgi/doi/10.1101/gr.200535.115. Freely available online through the Genome Research Open Access option. regulatory activity can predict the gain of function, allowing follow-up consideration of the site.

In recent years, artificial neural networks with many stacked layers have achieved breakthrough advances on benchmark data sets in image analysis (Krizhevsky et al. 2012) and natural language processing (Collobert et al. 2011). Rather than choose features manually or in a preprocessing step, convolutional neural networks (CNNs) adaptively learn them from the data during training. They apply nonlinear transformations to map input data to informative high-dimensional representations that trivialize classification or regression (Bengio et al. 2013). Early applications of CNNs to DNA sequence analysis surpass more established algorithms, such as support vector machines or random forests, at predicting protein binding and accessibility from DNA sequence (Alipanahi et al. 2015; Zhou and Troyanskaya 2015). More accurate models can more precisely dissect regulatory sequences, thus improving noncoding variant interpretation. However, to fully exploit the value of these models, it is essential that they are technically and conceptually accessible to the researchers who can take advantage of their potential.

Here, we introduce Basset, an open source package to apply deep CNNs to learn functional activities of DNA sequences. We used Basset to simultaneously predict the accessibility of DNA sequences in 164 cell types mapped by DNase-seq by the ENCODE Project Consortium and Roadmap Epigenomics Consortium (The ENCODE Project Consortium 2012; Roadmap Epigenomics Consortium et al. 2015). From these data sets, CNNs simultaneously learn the relevant sequence motifs and the regulatory logic with which they are combined to determine cell-specific DNA accessibility. We show that a model achieving this level of accuracy provides meaningful, nucleotide-precision measurements. Subsequently, we assign Genome-wide association study (GWAS) variants cell-type-specific scores that reflect the accessibility

(C) 2016 Kelley et al. This article, published in Genome Research, is available under a Creative Commons License (Attribution 4.0 International), as described at http://creativecommons.org/licenses/by/4.0/. 
difference predicted by the model between the two alleles. These scores are highly predictive of the causal SNP among sets of linked variants. Importantly, Basset puts CNNs in the hands of the genome biology community, providing tools and strategies for researchers to train and analyze models on new data sets. In conjunction with genomic big data, Basset offers a promising future for understanding how the genome crafts phenotypes.

\section{Results}

\section{Deep CNNs predict genome accessibility}

DNA sequence codes for the chromatin shifts that transform cells in development and disease. We focused here on DNA accessibility due to the abundance of available data and significant association with conserved segments (Thurman et al. 2012), disease variation (Maurano et al. 2012), and eQTLs (Degner et al. 2012). The ENCODE Project Consortium performed DNase-seq on 125 cell types (Thurman et al. 2012), and the Roadmap Epigenomics Consortium curated an additional 39 (Roadmap Epigenomics Consortium et al. 2015). We collected and merged these sets, resulting in 2 million sites across all cells (Methods; Fig. 2A below). The GENCODE v18 reference gene catalog annotates these sites as $17 \%$ promoters, $47 \%$ intragenic, and $36 \%$ intergenic. A minority, $4.1 \%-19.0 \%$ (median $8.2 \%$ ), are accessible in any individual cell type, with $3.8 \%$ constitutively open in $>50 \%$ of the cells. For each DNase I hypersensitive site (DHS), we extracted $600 \mathrm{bp}$ from the hg19 reference genome around the midpoint as input to the model.

To learn the DNA sequence signals of open versus closed chromatin in these cells, we applied a deep CNN. CNNs have proven highly effective in a number of diverse tasks; this set recently includes biological sequence analysis (Alipanahi et al. 2015; Zhou and Troyanskaya 2015). As opposed to manually specifying features or performing a preprocessing step to statistically learn them, CNNs perform adaptive feature extraction to map input data to informative representations during training. The convolution operation is the engine of the CNN. In a convolution layer, the algorithm scans a set of weight matrices called filters across the input; these weight matrices learn to recognize relevant patterns (Fig. 1). Prior work has demonstrated that with a sufficiently large data set, deep neural networks can learn far more expressive and accurate models than other common approaches like random forests or kernel methods (Bengio et al. 2013).

For DNA sequences, the initial convolution layer corresponds to optimizing the weights of a set of position weight matrices (PWMs), which are a well-studied tool in bioinformatics (Stormo 2000). These PWM filters search for their motifs along the sequence and output a matrix with a row for every filter and column for every position in the sequence (Fig. 1). Computing nonlinear functions of the information flowing through the network allows for more expressive models. In each convolution layer, we apply a rectifier operation (i.e., set negative values to zero) to the matrix of filter output (Nair and Hinton 2010). Finally, we pool adjacent values by taking the maximum in a small window. This operation reduces the dimension of the input to the next layer (and thus the computation required in training). It also provides invariance to small sequence shifts to the left or right.

Subsequent convolutional layers operate analogously on the output of the prior layer. Thus, for DNA sequences, they capture spatial interactions between the initial PWM filter outputs. The full architecture of our neural network includes three convolution
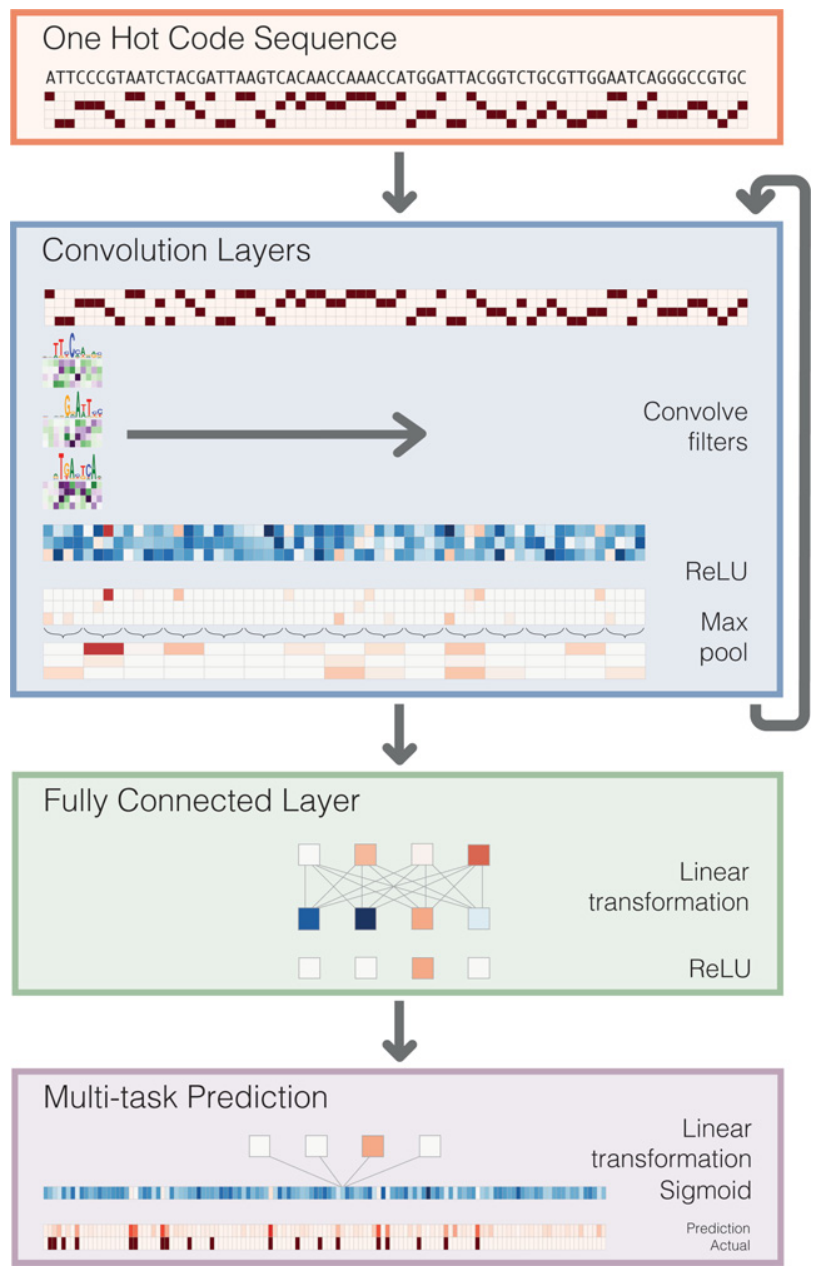

Figure 1. Deep convolutional neural network (CNN) for DNA sequence analysis. Basset predicts the cell-specific functional activity (here DNase I hypersensitivity) of sequences. First, we convert the sequence to a "one hot code" representation, where each position has a four-element vector with one nucleotide's bit set to one. Convolution layers proceed by scanning weight matrices across the input matrix to produce an output matrix with a row for every convolution filter and a column for every position in the input (minus the width of the filter). We apply a rectified linear unit (ReLU) nonlinear transformation to the convolution output and pool by taking the maximum across a window of adjacent positions. The first convolution layer operates directly on the one hot coding of the input sequence, making the convolution filters akin to the common bioinformatics tool position weight matrices. Subsequent convolution layers consider the orientations and spatial distances between patterns recognized in the previous layer. Fully connected layers perform a linear transformation of the input vector and apply a ReLU. The final layer performs a linear transformation to a vector of 164 elements that represents the target cells. A sigmoid nonlinearity maps this vector to the range zero to one, where the elements serve as probability predictions of DNase I hypersensitivity, to be compared via a loss function to the true hypersensitivity vector.

layers and two layers of fully connected hidden nodes. In general, deeper networks are able to learn more abstract representations; here, depth allows the algorithm to consider the sequence at multiple resolutions and learn sophisticated regulatory codes that combine the recognized sequence motifs. The final layer outputs 164 predictions for the probability that the sequence is accessible in each of the 164 cell types. During training, we compare these predictions to the experimentally measured accessibilities and update the model parameters to improve the predictions (see Methods). 
A

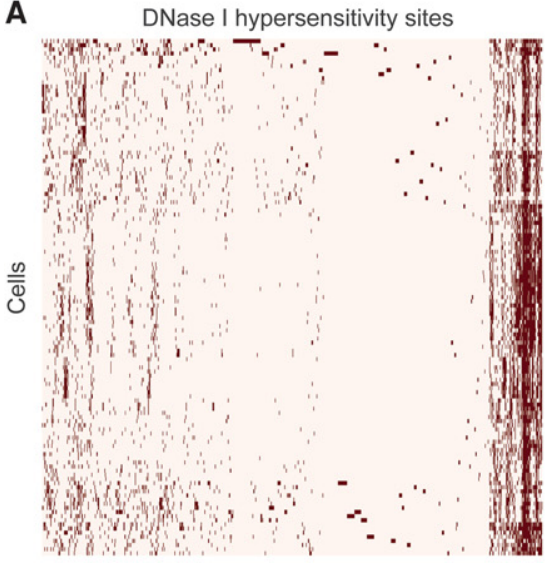

B

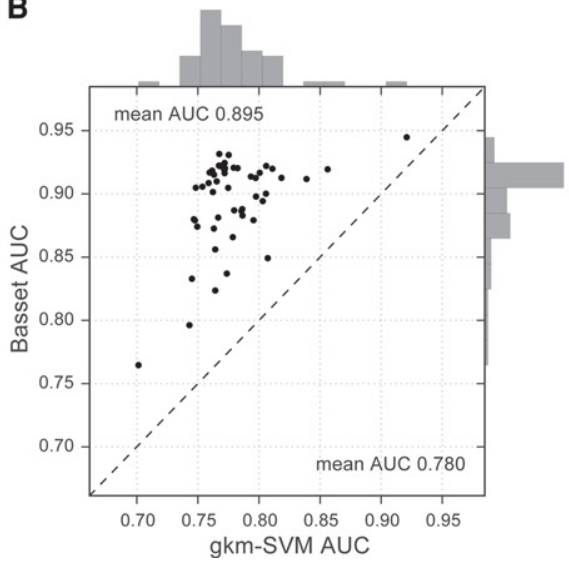

C

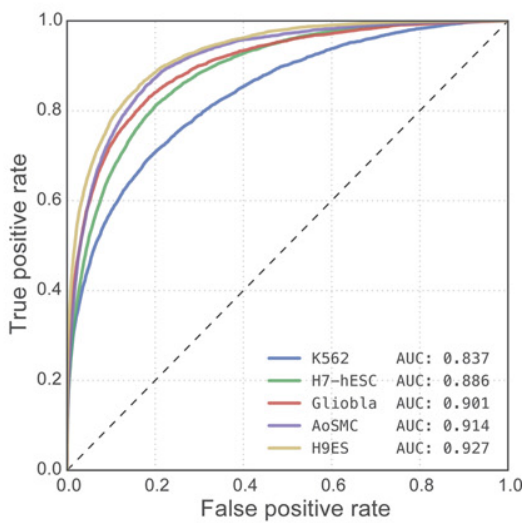

Figure 2. Basset accurately predicts cell-specific DNA accessibility. (A) The heat map displays hypersensitivity of 2 million DNase I hypersensitive sites (DHSs) mapped across 164 cell types. We performed average linkage hierarchical clustering using Euclidean distance to both cells and sites. (B) The scatter plot displays AUC for 50 randomly selected cell types achieved by Basset and the state-of-the-art approach gkm-SVM, which uses support vector machines. (C) The ROC curves display the Basset false-positive rate versus true-positive rate for five cells, selected to represent the $0.05,0.33,0.50,0.67$, and 0.95 quantiles of the AUC distribution.

We have released open source software implementing all procedures described in this paper, including routines to preprocess common functional genomics data, train the network, and extract the knowledge it has learned. We have named the package Basset, as an allusion to the extraordinary abilities of these hound dogs to learn a scent that they are subsequently able to detect and pursue.

We trained Basset and a recently published advance based on gapped $k$-mer support vector machines called gkm-SVM (Lee et al. 2015) to predict the accessibility of a set of test sequences in 164 cell types (see Methods). To synthesize sensitivity and specificity, which inherently trade off, we assessed the models using the area under the receiver operating characteristic curve (AUC), which plots the false-positive rate versus the true-positive rate. By this measure, Basset is more accurate than gkm-SVM, achieving a mean AUC of 0.895 over all cells, relative to 0.780 for gkm-SVM (Fig. 2B). Basset substantially improved the AUC for every cell type. At a false-positive rate of 10\%, Basset identifies 55\%-80\% of true-positive DHS sequences (Fig. 2C).

In imbalanced data sets like these, the area under the precision-recall curve (AUPRC) is also instructive. Basset achieves a greater mean AUPRC of 0.561 versus 0.322 for gkm-SVM (Supplemental Fig. S1). At a false-discovery rate of $20 \%$, Basset recalls $20 \%$ $35 \%$ of accessible sites. Thus, although Basset extracts substantial information about how sequence determines accessibility, no tool is presently accurate enough to annotate large genomes de novo.

In addition to predicting constitutive sites, Basset effectively captures cell- and lineage-specific accessibility (Supplemental Fig. S2). Cell- and lineage-specific sites are more challenging to predict, and accuracy computed on sites active in less than half the cells decreases to 0.858 AUC (Supplemental Fig. S3). Finally, Basset predicted accessibility of sites assigned to various annotation classes with consistent accuracy: 0.900 for promoters, 0.884 for intragenic, and 0.891 for intergenic (Supplemental Fig. S4).

\section{Basset recovers known protein binding motifs}

Though deep neural networks predict accurately, the principles that they learn are not trivially interpretable as they are in simpler linear models. However, substantial information can be extracted from the model by examining its parameters, modulating the flow of information through components of the network, and exploring its predictions on purposefully chosen sequences.

The typical DHS is a nucleosome-free region where protein(s) bind the DNA to create an accessible site (Sherwood et al. 2014; Voss and Hager 2014). Thus, we expect that a predictive model of accessibility will capture this dependence by learning the DNA binding sites of a variety of universal and cell-specific proteins. The first convolution layer of the model scans the DNA sequence with a set of pattern-recognizing filters whose weights are optimized during training. These filters are ideal for capturing this protein binding information.

The model's 300 convolution filters recovered an extensive repertoire of known DNA binding protein motifs (Fig. 3B). To aid in their interpretation, we nullified each filter by setting its output to a constant value: its mean output over all nucleotides in the test set. This obstructs the filter from passing any information forward through the network. We considered the vector containing the change in predicted accessibility in each cell type and quantified each filter's influence as this vector's sum of squares. Complementing that filter-centric analysis, we performed a protein-centric analysis by introducing known protein binding motifs from the CIS-BP database into the center of many sequences and measuring the change in predicted accessibility (Weirauch et al. 2014).

The critical genome architecture protein CTCF was most predictive of accessibility across all cell types. The model dedicates the most filters (12) to comprehensively represent CTCF's 19-bp-long DNA recognition site (Supplemental Fig. S5). Each filter focused on overlapping portions and variations of the motif. The AP-1 complex, consisting of proteins from the JUN, FOS, ATF, and JDP families, also emerged as highly influential via four filters. AP-1's important role in regulating open chromatin has been previously observed (Biddie et al. 2011).

At a $q$-value threshold of $0.1,45 \%$ of the filters aligned significantly to protein motifs in CIS-BP, which were originally acquired by independent ChIP-seq or in vitro experiments (Gupta et al. 2007; Weirauch et al. 2014). This set included highly similar

\section{Genome Research}

www.genome.org 
A

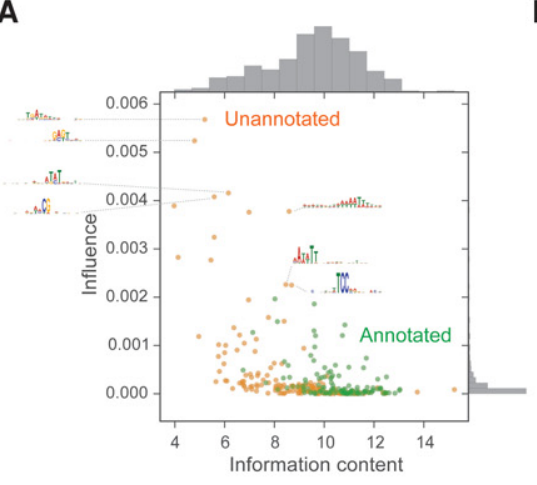

B
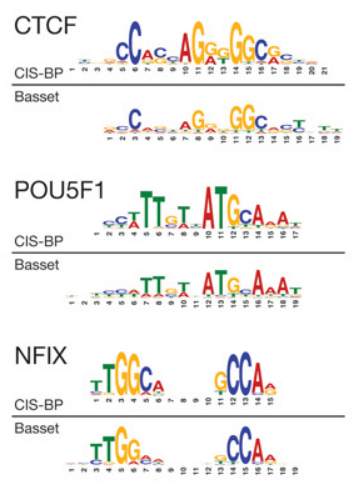
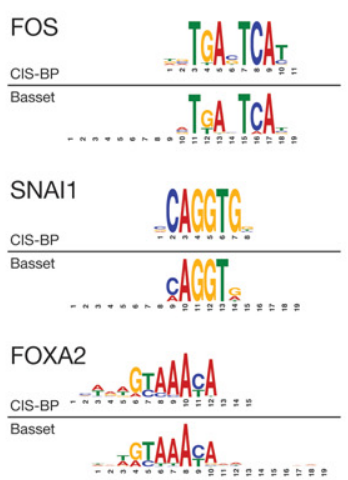
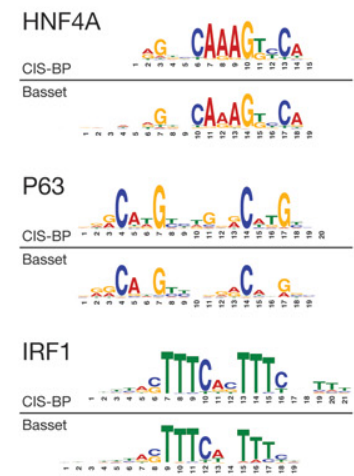

C

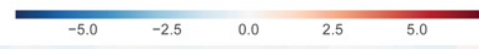

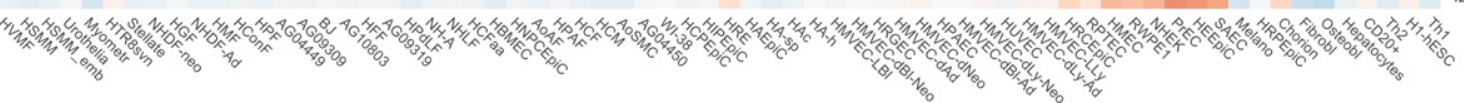

Figure 3. Basset initial convolutional layer discovers known and novel sequence motifs. $(A)$ In the scatter plot, the $x$-axis describes the information content for the PWMs represented by the 300 first layer convolution filters (Methods). The $y$-axis describes an influence score, which we compute by setting all output from the filter to its mean (thus nullifying the filter) and taking the sum of squares of the vector of accessibility prediction changes over all cells. We colored filters by whether or not they could be annotated at a q-value threshold of 0.1 by the TomTom motif comparison tool to known TF motifs in the human CIS-BP database. (B) Overall, $45 \%$ of filters could be annotated, including the alignments shown here. (C) Clustering the filters by their influence on accessibility predictions in each cell type revealed this set matching TP63, GRHL1, and KLF factors, which are known to be involved in epithelial development.

weight matrices for many developmental regulators; Figure 3B depicts a sample. Many more filters capture partial coverage of known motifs, which were deemed insignificant matches to the database after multiple testing correction (Supplemental Fig. S5). Motifs known to allow for variable spacing between two components were not prominently recognized by these partial motif filters (Reid et al. 2010).

Some unrecognized filters captured lower-order sequence composition, such as the known enrichment of higher GC content in TF-bound DHSs (Wang et al. 2012). One filter directly detects CpG's, which can be methylated and are a well-studied feature of regulatory modules (Bird and Wolffe 1999). Other examples measure poly-AT stretches and nonconsecutive C's and G's (thus avoiding the $\mathrm{CpG}$ ). Their influence emphasizes the importance of the local sequence context of binding motifs to their function (Fig. 3A; Levo and Segal 2014; Dror et al. 2015).

To further explore how these filters influence predictions, we studied the AP-1 consensus motif TGASTCA in detail. Rather than indiscriminately predicting accessibility upon detection of the motif, Basset accurately captures the nuance of these binding events. The model achieved an average AUC of 0.86 across all cell types on test sequences that contain the motif in the center $50 \mathrm{nt}$; a classifier that had only learned the motif would merely be guessing at which specific motif instances were bound and achieve AUC 0.5 .

To determine what features the model uses to vary its predictions for AP-1 motifs, we artificially inserted the motif into the center of a random set of sequences and compared predictions before and after. We focused the analysis on mammary fibroblast (HMF) and H7-hESCs, which serve as examples of cells that respond with strong and medium strength to the AP-1 motif, respectively. The motif alone was insufficient to predict high accessibility but does shift most predictions upward to varying degrees (Supplemental Fig. S6). We noted several features that influence Basset's predictions. The first nucleotide $5^{\prime}$ prefers [ACG] rather than T, and the first nucleotide $3^{\prime}$ prefers [CGT] rather than A. This is apparent in the weight matrix for the primary filter used by the model to identify AP-1 (Supplemental Fig. S7). In HMFs, the average prediction for the full consensus motif is 0.49 versus 0.25 for the motif with a $5^{\prime} \mathrm{T}$ and versus 0.10 for the motif with a $5^{\prime} \mathrm{T}$ and $3^{\prime} \mathrm{A}$.

The sequence composition of an additional $\sim 100 \mathrm{nt}$ of flanking sequence also influences the predictions. The clearest effect comes from flanking poly-AT stretches that drive down the prediction (Supplemental Fig. S7). So-called A-tracts are known to narrow the minor groove of the DNA double helix (Rohs et al. 2009). Additional protein binding sites also influence predictions. We observed an interesting case of a GGAART motif (best represented in CIS-BP by ETS family member FEV) that can overlap $5^{\prime}$ the AP-1 motif for a high prediction (Supplemental Fig. S8). We also observed that nearby weak nonconsensus TTASTCA AP-1 motifs increase the prediction (Supplemental Fig. S8). These observations suggest that CNNs offer a simple and effective approach to automatically capture the subtle influences on functional activity provided by local sequence composition (Slattery et al. 2014).

Several unrecognized filters had high information content, which indicates that they may refer to unannotated proteins or alternative binding modes of annotated ones. To further explore these filters, we computed the influence of nullifying each filter on the downstream cell accessibility predictions (Supplemental 
Fig. S9). Clustering these influence profiles reveals modules of filters matching proteins known to regulate development to their active cell types. For example, filters matching database motifs for known epithelial regulators TP63, GRHL1, and KLF factors are predictive of accessibility in a variety of epithelial cells (Fig. 3C; Wilanowski et al. 2008; Ray and Pollard 2012; Pignon et al. 2013). The unrecognized filters span a range of cell preferences, and future work will be necessary to determine their role and potential binding proteins.

In silico saturation mutagenesis pinpoints nucleotides driving accessibility

A trained model can be used to predict the functional activity of arbitrary sequences, offering a powerful approach to understand and apply the regulatory grammar that it has learned. Saturation mutagenesis experiments, in which every mutation to a sequence is tested, are a powerful tool for dissecting the exact nucleotides driving a functional activity (Patwardhan et al. 2009; Melnikov et al. 2012). State-of-the-art experimental approaches involve a complex procedure of synthesizing massive pools of oligonucleotides and measuring their activity in a parallel reporter assay (Melnikov et al. 2012; Patwardhan et al. 2012). By computing the predicted accessibility of all possible mutations to a sequence, Basset can be used to perform an in silico saturation mutagenesis.

We constructed heat maps that display the change in predicted accessibility from mutation at every position to each alternative nucleotide. These maps highlight the individual nucleotides most critical to a sequence's activity. We assigned two scores to every position: (1) The loss score measures the largest possible decrease and (2) the gain score measures the largest increase.

High loss scores mark positions with existing functional motifs where mutations can damage the motif and decrease accessibility. For example, the sequence mapped in Figure 4 is accessible in embryonic stem cells and contains the AP-1 motif. AP-1 complex members JUN and JUND ChIP-seq in H1-hESCs support the

A $118.436 \mathrm{mb}$ $118.437 \mathrm{mb}$
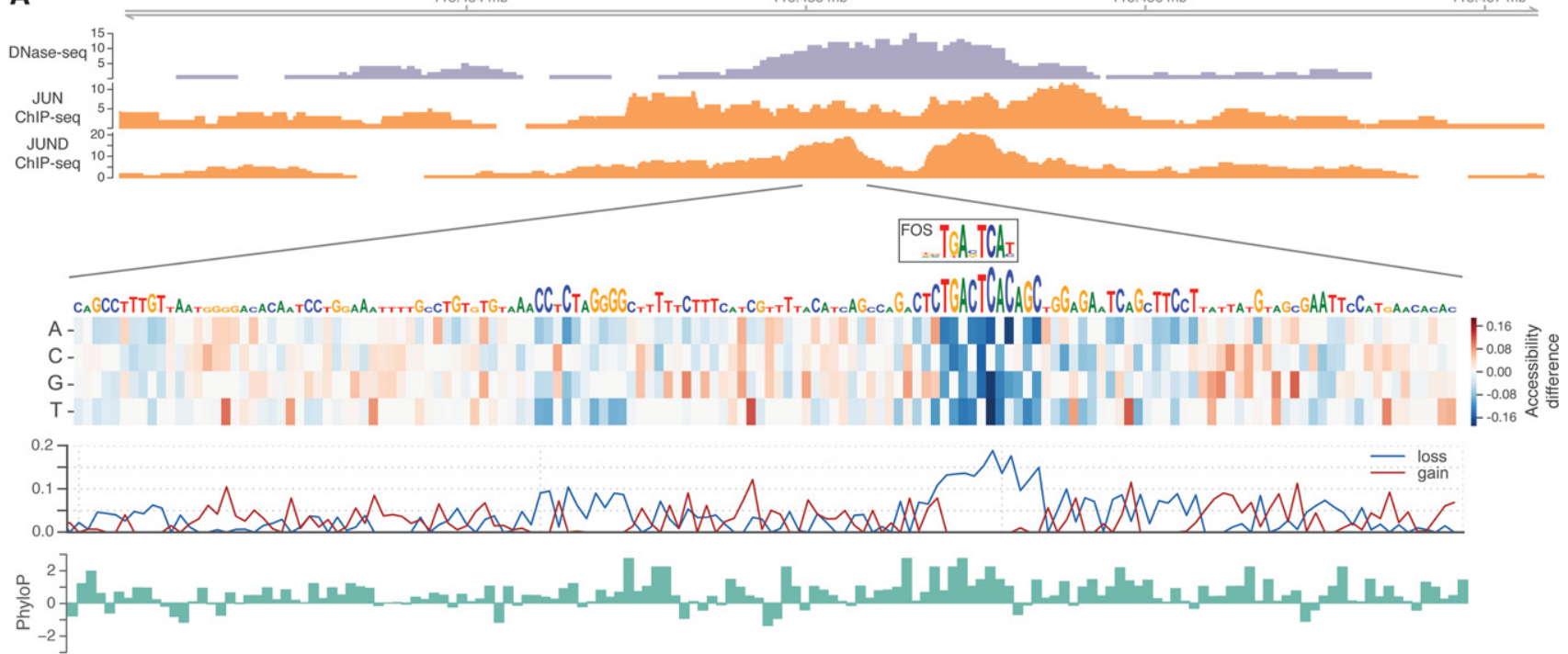

B

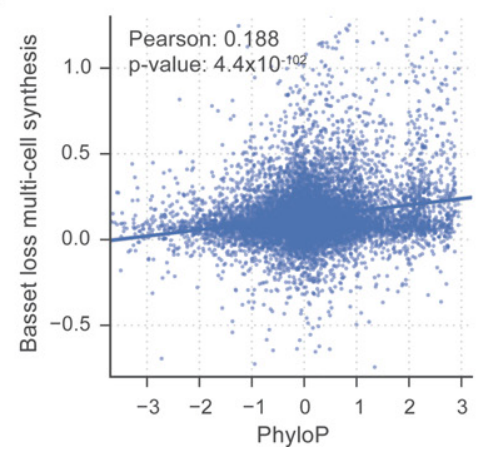

C

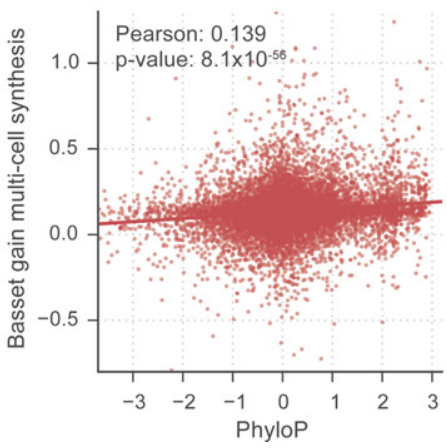

D

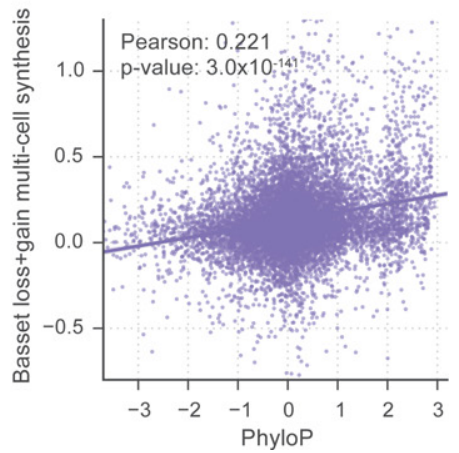

Figure 4. In silico saturated mutagenesis for DNase I hypersensitivity. (A) We used Basset to predict the effect of every mutation on the accessibility of the region Chr 9: 118,434,976-118,435,175 in H1-hESCs. The heat map displays the change in predicted accessibility for mutated sequences. Each column corresponds to a position in the sequence. Each row represents mutation to the corresponding nucleotide. In the line plot below, loss scores measure the maximum decrease among all mutations from the true nucleotide. Gain scores measure the maximum increase. We drew nucleotides to be proportional to the loss score, beyond a minimum height. At this locus, the model highlights the TGASTCA motif of the AP-1 complex (shown as the CIS-BP database motif for FOS). ChIP-seq of JUN and JUND in H1-hESCs confirm binding of the complex. The bound motif displays high conservation according to PhyloP. (B) Genome-wide, loss scores had a strong relationship with PhyloP (see Methods). (C,D) Gain scores alone had a weaker relationship (C), but the combination of gain and loss scores achieved the strongest relationship $(D)$.

\section{Genome Research}

www.genome.org 
binding event, and PhyloP conservation statistics support the precise relevance of the TGASTCA motif (Pollard et al. 2010). Mutations within the motif and numerous flanking nucleotides result in decreased predicted accessibility. Genome-wide, a synthesis of loss scores from all cell types significantly correlated with PhyloP (Pearson 0.188; P-value $4.4 \times 10^{-102}$ ) (see Methods; Fig. 4B).

In contrast, high gain scores suggest latent potential in a sequence; the corresponding mutation often introduces a functional motif to increase the predicted accessibility. Although such a position does not mark a present functional motif, there may be negative selection against one forming and rearranging accessibility in the region. In support of this effect, considering both loss and gain scores increased the Pearson correlation with PhyloP to $0.221\left(P\right.$-value $\left.3.0 \times 10^{-141}\right)$ (Fig. 4D). This correlation was consistent across promoter, intragenic, and intergenic annotation classes (Supplemental Fig. S10).

\section{Basset predicts greater accessibility changes for likely causal GWAS SNPs}

Genome-wide association studies (GWAS) have uncovered ample noncoding variants associated with physical traits and disease in human populations (Welter et al. 2014). DHSs are highly enriched for GWAS SNPs, which can modulate the accessibility of the site to affect local gene expression (Degner et al. 2012; Maurano et al. 2012). Basset captures the sequence signals driving accessibility and ought to have predictive power for prioritizing noncoding variants and suggesting mechanistic hypotheses for further investigation into their causal role for the phenotype. For this purpose, we defined SNP Accessibility Difference (SAD) profiles as the difference in predicted accessibility across cell types between two alleles.

The scarcity of confirmed positive examples of noncoding causal variants challenges a thorough assessment of the value of SAD scores for GWAS prioritization. Instead, we studied probabilistic assessments of causality assigned by an orthogonal method:
8741 GWAS SNPs associated with auto-immune disease were analyzed with a statistical method called PICS, which leverages dense genotyping data to assign a probability of being the causal SNP among a nearby set of SNPs in linkage disequilibrium (LD) (Farh et al. 2015). In a large number of cases, PICS identified the causal SNP with high probability.

We focused on a set of 7252 GWAS SNPs for which no SNP in LD affects a protein coding gene, and classified 235 high-PICS SNPs that were assigned causal probability of 0.5 or more and 3004 low-PICS SNPs that were assigned causal probability of 0.05 or less. SAD profile means were significantly greater for the set of high-PICS SNPs (Mann-Whitney $U$ test, $P$-value $<1.3 \times 10^{-7}$ ) (Supplemental Fig. S11). More than seven times more high-PICS SNPs than low were predicted to change accessibility by an average of more than 0.1 over the cell types (Fig. 5A). Coverage of the SNPs in this set is wide: $31 \%$ of all index SNPs had at least one SNP in its LD set for which the model predicted a $>10 \%$ change in probability of accessibility in some cell type (Supplemental Fig. S11). We report all predicted mutation effects on this data set in Supplemental Table S1.

Among the agreements with PICS was rs4409785, associated with vitiligo (Jin et al. 2012), rheumatoid arthritis (Okada et al. 2013), and immune mechanisms in multiple sclerosis (Sawcer et al. 2011). PICS assigned rs $440978585.3 \%$ probability of causality for vitiligo. The SNP is located in a 559-kb gene desert. However, it has been hypothesized to regulate $T Y R$, which although $6.28 \mathrm{Mb}$ away, offers a plausible mechanism for the skin color disease vitiligo. TYR catalyzes conversion of tyrosine to melanin, the pigment that gives skin its color (Jin et al. 2012).

Basset predictions support this hypothesis; the more prevalent $\mathrm{T}$ allele is devoid of activity, but the $\mathrm{C}$ allele creates a motif recognized by the model's CTCF filters (Fig. 5B). Although this sequence imperfectly matches the CTCF database motif, Basset predicts dramatically increased accessibility in all cell types, including an increase in H1-hESCs from $0.8 \%$ probability to $73.24 \%$. To assess experimental evidence for allele-specific CTCF
A

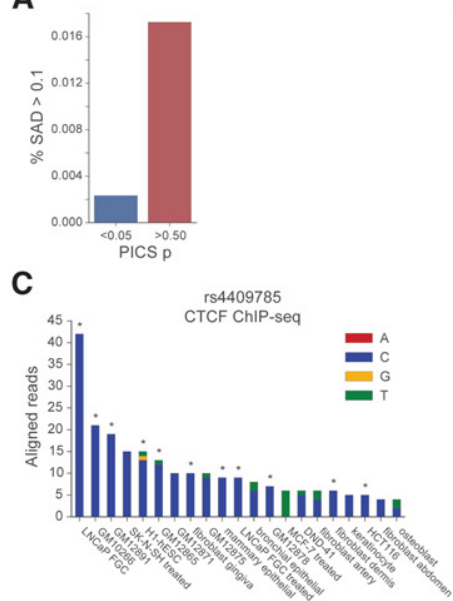

B

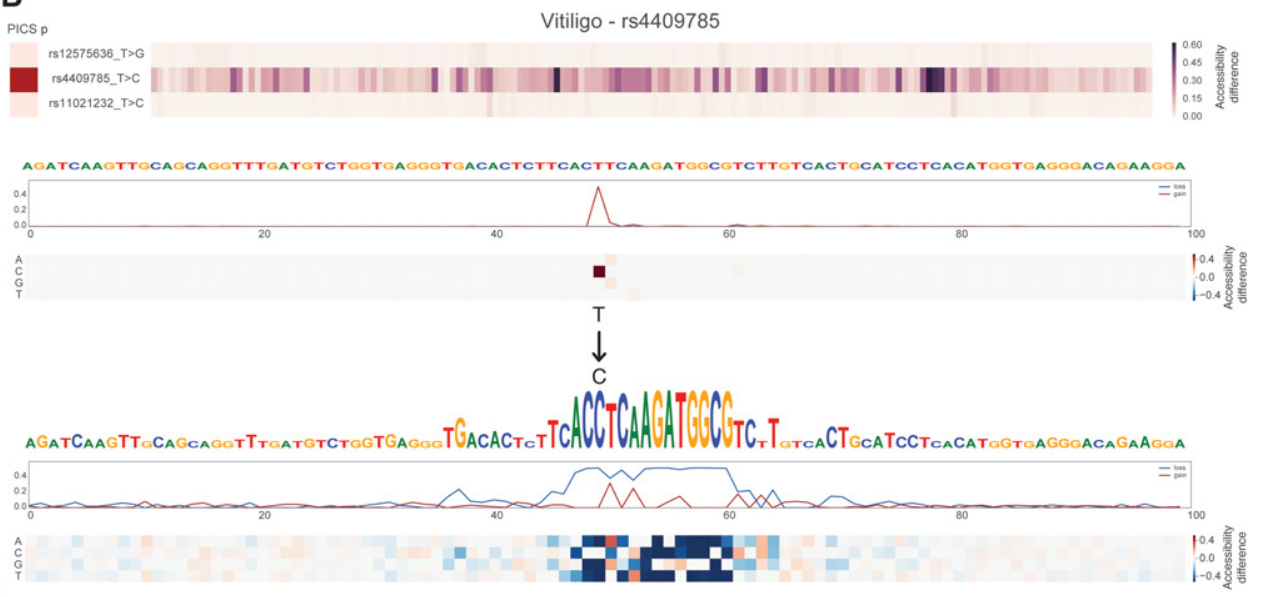

Figure 5. SNP accessibility difference (SAD) scores enable genomic variant interpretation. (A) Basset assigned greater scores to likely causal GWAS SNPs (PICS probability $>0.5$ ) versus unlikely nearby SNPs (PICS probability $<0.05$ ) as determined by population fine mapping data. The bars measure the proportion of SNPs assigned a SAD profile mean across all cell types of more than 0.1. (B) We annotated rs4409785 among the highest SAD scores, in agreement with the PICS view of this haplotype block. Basset predicts the more common T allele to be completely dormant, but the region transforms with the $C$ allele into a site deemed by Basset to have very high accessibility due to a CTCF binding site. (C) CTCF ChIP-seq in 88 unique cell types strongly supports the allele specificity of CTCF at this site. We plotted cells with more than three reads (summed across replicates) aligned to the site, and marked significant peak calls with asterisks. The 11 cells with significant peak calls all sequenced the $C$ allele. 
binding, we downloaded 88 unique CTCF ChIP-seq data sets performed in a variety of cell types by ENCODE (The ENCODE Project Consortium 2012). Of these, 21 cell types had more than three reads aligned to rs4409785, of which 11 had significant peak calls (Fig. 5C). Every significant peak sequenced the $\mathrm{C}$ allele, strongly supporting the allele specificity of CTCF binding at this site.

Recent work has demonstrated that modulating CTCF binding at topologically associating domain boundaries can dramatically affect gene expression by altering the architecture of the genomic region (Guo et al. 2015; Lupiáñez et al. 2015). By modeling the underlying sequence driving the experimental measurement, Basset's highlight of rs4409785 suggests a functional hypothesis to accelerate study of this widely influential variant.

\section{Leveraging large-scale models allows accurate and efficient prediction of new data sets}

To make the most of machine learning to identify causal SNPs, researchers must train models on data from the appropriate (perhaps specialized) cell type (Lee et al. 2015). In many cases, such cells may be experimentally characterized by individual laboratories rather than by large-scale mapping projects. We designed Basset to accommodate this frequent and critical scenario. We also hypothesized that Basset can rapidly learn to accurately predict new data by leveraging a model pretrained on public data.

To test this hypothesis, we removed 15 data sets spanning the range of AUC from the pool of 164 studied above. We retrained on the remaining 149 "public" data sets to establish a "pretrained" model. This model achieved an average AUC of 0.892 across the 149 cells, on par with the full model analyzed above.

For each remaining data set individually, we sampled a matching number of sites from the "public" 149 to serve as negative examples. Rather than train from scratch on the data set alone, we initialized the model parameters with those from the "pretrained" model (Methods). By providing this head start, we need only perform one training pass through the new data to achieve models with predictive accuracies rivaling those from the full 164 cell model above (Fig. 6; Supplemental Fig. S12; Supplemental Table S2). We completed this training procedure in an average of $6.5 \mathrm{~h}$ on a Macbook 2.8-GHz Intel core i7 or 18 min on an NVIDIA Tesla K20m GPU. Thus, this approach allows researchers to train highly accurate deep $\mathrm{CNN}$ models on common computer hardware in a few hours.

\section{Discussion}

In this work, we introduced Basset, an open source package to apply deep CNNs to learn DNA sequence activity. Basset effectively learned the complex code of DNA accessibility across many cell types and substantially surpassed the predictive accuracy of the present state of the art. We demonstrated how our model precisely implicates the nucleotides driving activity, highlighting genomic positions with either fragile activity that can be lost by mutation or latent potential activity that can be unlocked by mutation. These sites are more conserved, and their mutation is more likely to cause disease.

Predicting the functional output of DNA sequences is a fundamental problem in computational biology with a long history of approaches (Bussemaker et al. 2001; Segal et al. 2003; Beer and Tavazoie 2004). Our work, considered with independent successes in other groups (Alipanahi et al. 2015; Zhou and Troyanskaya 2015), indicates that hardware (GPGPUs), software (CNNs), and training data (mammalian genome-wide) have now converged to enable drastically enhanced performance on such problems. What has been missing from our previous models? One primary difference is that CNNs naturally consider positional relationships between sequence signals. In contrast, most sequence kernel approaches immediately throw away position information to represent DNA as vectors of $k$-mer counts (Ghandi et al. 2014). Position-specific sequence kernels exist, but they greatly increase the dimensionality of the raw input space to which the sequence is initially mapped. Unfortunately, such kernel methods do not scale well to higher dimensional inputs, both in computational efficiency and in predictive performance, due to the curse of dimensionality: Generalization via interpolation suffers in higher

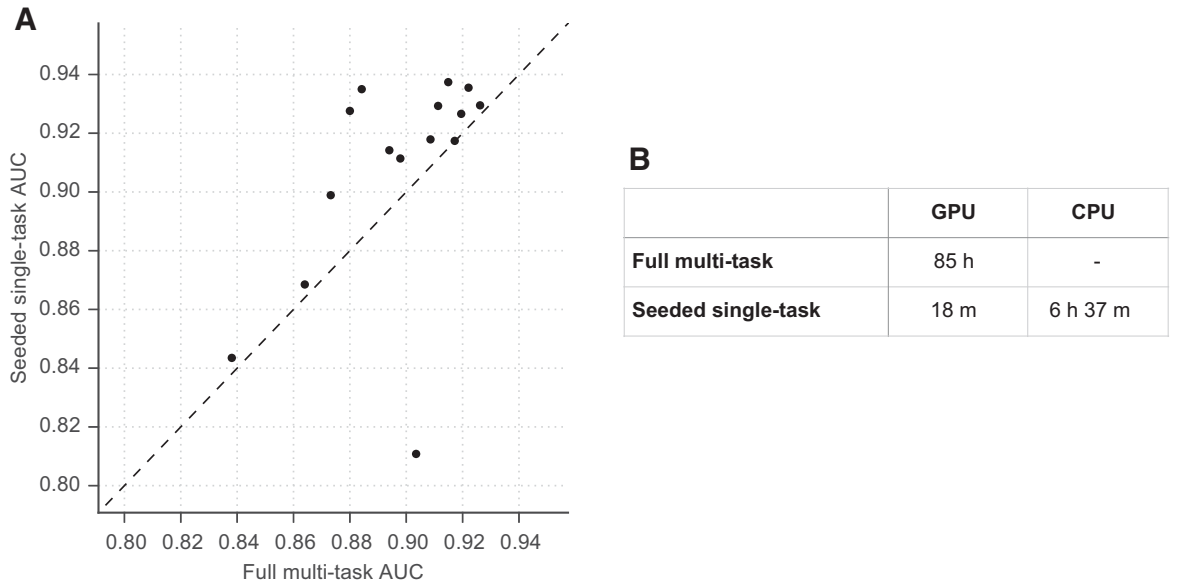

Figure 6. Basset leverages large-scale public data to inform additional data set learning. ( $A$ ) The scatter plot shows AUC for 15 data sets achieved by the full model trained on all 164 cell types on the $x$-axis and AUC achieved by a procedure to simulate studying that data set alone on the $y$-axis. To study the data set alone, we pretrain a model on 149 cells (after removing these 15), seed training of the additional cell with that model's parameters, and perform a single training pass through the new data. This rapid procedure was effective for all but one data set (HRCEpiC, renal cortical epithelial cells), for which multitask training with the many other similar epithelial cells was beneficial. The AUC improvement for many cells suggests that our full model may benefit from increased capacity or decreased regularization. (B) The seeded training procedure is far faster on the GPU and allows for feasible CPU training.

\section{Genome Research}

www.genome.org 
dimensional spaces. By jointly learning a meaningful representation and a smooth parameterized projection to the outputs, CNNs, in essence, learn the kernel. Finally, neural networks trained via stochastic gradient descent scale very well to large data sets, allowing us to learn good parameters within a general and expressive model structure.

The most successful prior approaches to analyze noncoding variants compare them to the broad regions that functional genomics experiments have annotated to have reproducible accessibility, protein binding, and/or histone modifications (Fu et al. 2014; Kircher et al. 2014; Ritchie et al. 2014). Basset has two primary benefits over this approach. By directly modeling the mapping from sequence to activity, Basset implicates the precise nucleotides influencing accessibility, providing a finer-resolution view than mere overlap with a broad region. Basset assigns low SAD profiles to many nucleotides overlapped by these regions, calling into question their consideration for causal roles.

Furthermore, if the genome sequenced in the original experiment included only the inaccessible allele, there will be no indication that accessibility is relevant to the SNP. Basset readily identifies these gain-of-function mutations, as demonstrated for CTCF binding to rs4409785 (Fig. 5). As statistical searches for influential variants in human populations continue, rare variants will make up a greater proportion. Especially for these rare variants, the functional genomics experiment will be unlikely to have been performed in the necessary genetic background. This growing trend makes methods like Basset even more important if we are to interpret these variants.

With Basset, a researcher can perform a single sequencing assay in their cell type of interest and simultaneously learn that cell's chromatin accessibility code and annotate every mutation in the genome with its influence on present accessibility and latent potential for accessibility. By leveraging large-scale public data, one can train accurate models on common computational hardware. Researchers continue to discover noncoding variants in human populations that influence phenotypes, and such annotation will be indispensable for interpreting how those variants function. As the tide of functional genomics data continues to flow, novel machine learning approaches such as deep CNNs have great power to aid this goal.

\section{Methods}

\section{DNase I hypersensitivity data}

We downloaded DNase-seq peak BED format files for 125 cell types from the ENCODE Project Consortium (2012) and 39 cell types from the Roadmap Epigenomics Consortium (2015). For both sets, the previous groups called peaks using the HotSpot algorithm and performed a simulation procedure to establish a set with 1\% false-discovery rate. We considered all peaks, regardless of overlap with genomic annotations.

To merge the peaks into one set, we first extended each one from its midpoint to $600 \mathrm{bp}$. We greedily merged peaks based on their distance to an adjacent peak until no peaks overlapped by $>200 \mathrm{bp}$. During a merger of peaks, we specified the activity of the new peak as the union of the sets of active cell types for each individual peak. We specified the new limits by extending from a weighted average of the two peak midpoints, weighted by the number of cells each individual peak was active in. This produced a set of 2,071,886 peaks, of which $4.1 \%-19.0 \%$ (median $8.2 \%$ ) were active in the individual cell types. We extracted the hg19 reference genome sequence for each merged site as input to the mod- el. Thus, the input data to training for each site include its 600-bp DNA sequence and a binary vector to indicate the presence of a significant peak in each of the 164 cell types.

For some analyses of model predictions, we divided sites among promoter (within $2 \mathrm{~kb}$ of a transcription start site), intragenic (overlapping a gene's span), and intergenic classes using the GENCODE v18 reference catalog (Harrow et al. 2012).

\section{Deep CNN}

Deep CNNs are a type of deep neural network that are specifically parameterized to take advantage of known spatial structure. They were originally developed to recognize handwritten digits in images (LeCun et al. 1998). Convolutional networks have since become the gold standard for numerous image analysis tasks (Krizhevsky et al. 2012; Szegedy et al. 2015). Recently, convolutional networks have been modified for use within natural language processing and text analysis by applying a one-dimensional convolution temporally over a sequence (Hu et al. 2014; Zhang et al. 2015).

We implemented a deep CNN using Torch7 (http://torch.ch). Initially, we map the DNA sequence to four rows of binary variables representing the presence or absence of an A, C, G, or T at each nucleotide position. The first convolutional layer of the network scans PWMs across the sequence (Fig. 1). The matrix weights are parameters learned from the data. These are typically referred to as filters in the CNN literature. After convolving the matrix across the sequence, we applied a rectified linear ReLU nonlinearity $[f(x)=\max (0, x)]$, which has been found helpful in avoiding the vanishing gradient problem that plagued early deep learning research (LeCun et al. 1998; Nair and Hinton 2010). Finally, we "pool" adjacent positions by taking the maximum from a small window in order to reduce the number of parameters and achieve invariance to small shifts of the sequence left or right.

Subsequent convolutional layers operate on the output of the prior layer, which represents recognition of filter patterns across windows of the sequence. After three convolutional layers, we placed two standard, fully connected artificial neural network hidden layers and a final fully connected sigmoid transformation to 164 outputs, representing the predicted probability of accessibility in each cell type. We trained to minimize the binary cross entropy loss function, summed over these 164 outputs.

We applied stochastic gradient descent to learn all model parameters, including those representing convolution filters, using RMSprop updates on minibatches (Tieleman and Hinton 2012). First, we randomly initialized the parameters to small values. During training, the network computes predictions for small batches of sequences. We compare these predictions to the true experimental measurements using the loss function. We then update the model parameters to improve those predictions by taking a step in the direction of the gradient of the parameters with respect to the loss function, which we compute using the back propagation algorithm. After iterating over many batches of training data, the model begins to recognize specific sequence motifs indicative of accessibility and to project this recognition through the network to the cell predictions. We continue training until accuracy ceases to increase on a held-out validation set for 12 passes through the training data.

The user must specify the number of each type of layer, number of filters per convolution layer, filter sizes, pooling widths, fully connected layer units, and numerous regularization and training optimization parameters. We experimented with various model architectures and hyperparameter settings using Bayesian optimization, implemented in the package Spearmint (available from https ://github.com/HIPS/Spearmint) (Snoek et al. 2012). We committed to analyzing a top-performing architecture that is depicted in 
Supplemental Figure S13. Importantly, we apply batch normalization after every layer, which substantially stabilized training optimization (Ioffe and Szegedy 2015).

\section{Training, validation, and test data sets}

From the 2,071,886 total sites, we randomly reserved 71,886 for testing and 70,000 for validation, leaving 1,930,000 for training. We trained and tested on all cell types. We performed optimization directly on the training set. We used the validation set for "early stopping" after 12 epochs of unimproved validation loss and Bayesian optimization. We performed all assessment and analysis on the test set.

\section{gkm-SVM}

We downloaded gkm-SVM v1.3 from http://www.beerlab.org/ gkmsvm/ (Ghandi et al. 2014; Lee et al. 2015). Because the code computes the full Gram matrix, we could only feasibly train on a 100,000 subsample of the full data set. For each cell type, we down-sampled the inactive sequences to match the number of active sequences. Though Basset easily handles imbalanced data sets, we found the natural imbalances of this DHS data set significantly decreased gkm-SVM accuracy. When using default options, gkmSVM required $16 \mathrm{~d}$ to train and test on 50 randomly selected cell types.

\section{Motif analysis}

We converted Basset-learned first convolution layer filters to probabilistic PWMs by counting nucleotide occurrences in the set of sequences that activate the filter to a value that is more than half of its maximum value. We identified the likely binding protein for the motifs by querying the CIS-BP database (accessed on June 12,2015 ) (Weirauch et al. 2014) using the TomTom v4.10.1 search tool (Gupta et al. 2007) and requiring an FDR $q$-value $<0.1$. We computed the information content for a motif as

$$
\mathrm{IC}=-\sum_{i, j} b_{j} \log _{2}\left(b_{j}\right)+\sum_{i, j} m_{i j} \log _{2}\left(m_{i j}\right),
$$

where $m$ is the $19 \times 4$ matrix of nucleotide probabilities for the motif, and $b$ is the length 4 array of background hg19 nucleotide probabilities.

\section{Comparison of Basset predictions to PhyloP}

We used Basset to compute loss and gain scores for every nucleotide. We compute the loss score as the predicted activity with the reference nucleotide subtracted by the minimum predicted activity after mutating the position to the alternative 3 nucleotides (nt). We compute the gain score as the maximum predicted activity after mutation subtracted by the reference nucleotide activity. To ask whether these scores have a significant statistical relationship with nucleotide conservation, we required a method to consider all 328 scores per nucleotide with respect to PhyloP. We applied a linear regression model with ridge penalty, training on $80 \%$ of the nucleotides and testing on the remaining $20 \%$. We limited the analysis to nonrepetitive regions where more-confident PhyloP statistics can be assigned and to the center $100 \mathrm{bp}$ of the DHSs, where Basset makes stronger predictions (Supplemental Fig. S14).

\section{PICS}

We downloaded 8741 PICS SNP annotations from the supplement of the investigators' manuscript (Farh et al. 2015). For SAD profile comparison, we focused on an unquestionably noncoding set of 7252 SNPS by removing all SNPs linked to a SNP in a protein cod- ing gene. Without sufficient training data to learn weights for the various cell types studied, we resorted to comparing the SAD profile means to the PICS causal SNP probabilities.

\section{Seeding with pretraining model parameters}

To estimate Basset's accuracy on additional novel data sets, we removed 15 of 164 from the full set and trained a model on the remainder, which we refer to as "public" in order to simulate a future scenario where one might leverage existing public data sets to make better predictions on a new data set. For each of the 15 leftout data sets, we seeded the model with the parameters from the "public" model. We replaced the final hidden layer of the model to make predictions for the single new target and initialized the new parameters as above. Finally, we performed a single pass through the data with a halved learning rate for the stochastic gradient descent optimization. Additional passes through the data overfit this smaller prediction task.

\section{Software availability}

Source code implementing all steps-data preprocessing, training, and downstream analysis-is available in the package Basset from http://www.github.com/davek44/Basset. In addition, source code is included in the Supplemental Material.

\section{Acknowledgments}

We thank Abbie Groff, David Hendrickson, Marta Mele, Stephanie Fine Sasse, Chinmay Shukla, and Michael Ziller for feedback on the manuscript. D.R.K. was supported by National Institute of Environmental Health Sciences (NIH) K25 award ES022984-03. J.L.R. was supported by National Institute of Mental Health (NIH) grant R01MH102416-03.

\section{References}

Alipanahi B, Delong A, Weirauch MT, Frey BJ. 2015. Predicting the sequence specificities of DNA- and RNA-binding proteins by deep learning. Nat Biotechnol 33: 831-838.

Arnold P, Schöler A, Pachkov M, Balwierz PJ, Jørgensen H, Stadler MB, van Nimwegen E, Schübeler D. 2013. Modeling of epigenome dynamics identifies transcription factors that mediate Polycomb targeting. Genome Res 23: 60-73.

Beer MA, Tavazoie S. 2004. Predicting gene expression from sequence. Cell 117: $185-198$.

Bengio Y, Courville A, Vincent P. 2013. Representation learning: a review and new perspectives. IEEE Trans Pattern Anal Mach Intell 35: 1798-1828.

Benveniste D, Sonntag H-J, Sanguinetti G, Sproul D. 2014. Transcription factor binding predicts histone modifications in human cell lines. Proc Natl Acad Sci 111: 13367-13372.

Biddie SC, John S, Sabo PJ, Thurman RE, Johnson TA, Schiltz RL, Miranda TB, Sung M-H, Trump S, Lightman SL, et al. 2011. Transcription factor AP1 potentiates chromatin accessibility and glucocorticoid receptor binding. Mol Cell 43: 145-155.

Bird AP, Wolffe AP. 1999. Methylation-induced repression: belts, braces, and chromatin. Cell 99: 451-454.

Bussemaker HJ, Li H, Siggia ED. 2001. Regulatory element detection using correlation with expression. Nat Genet 27: 167-174.

Collobert R, Weston J, Bottou L, Karlen M, Kavukcuoglu K, Kuksa P. 2011. Natural language processing (almost) from scratch. J Mach Learn Res 12: 2493-2537.

Das R, Dimitrova N, Xuan Z, Rollins RA, Haghighi F, Edwards JR, Ju J, Bestor TH, Zhang MQ. 2006. Computational prediction of methylation status in human genomic sequences. Proc Natl Acad Sci 103: 10713-10716.

Degner JF, Pai AA, Pique-Regi R, Veyrieras J-B, Gaffney DJ, Pickrell JK, De Leon S, Michelini K, Lewellen N, Crawford GE, et al. 2012. DNaseI sensitivity QTLs are a major determinant of human expression variation. Nature 482: 390-394. 
Dror I, Golan T, Levy C, Rohs R, Mandel-Gutfreund Y. 2015. A widespread role of the motif environment in transcription factor binding across diverse protein families. Genome Res 25: 1268-1280.

The ENCODE Project Consortium. 2012. An integrated encyclopedia of DNA elements in the human genome. Nature 489: 57-74.

Farh KK-H, Marson A, Zhu J, Kleinewietfeld M, Housley WJ, Beik S, Shoresh N, Whitton H, Ryan RJH, Shishkin AA, et al. 2015. Genetic and epigenetic fine mapping of causal autoimmune disease variants. Nature 518: $337-343$.

Fu Y, Liu Z, Lou S, Bedford J, Mu XJ, Yip KY, Khurana E, Gerstein M. 2014. FunSeq2: a framework for prioritizing noncoding regulatory variants in cancer. Genome Biol 15: 480.

Ghandi M, Lee D, Mohammad-Noori M, Beer MA. 2014. Enhanced regulatory sequence prediction using gapped $k$-mer features. PLoS Comput Biol 10: e1003711.

Guo Y, Xu Q, Canzio D, Shou J, Li J, Gorkin DU, Jung I, Wu H, Zhai Y, Tang Y, et al. 2015. CRISPR inversion of CTCF sites alters genome topology and enhancer/promoter function. Cell 162: 900-910.

Gupta S, Stamatoyannopoulos JA, Bailey TL, Noble W. 2007. Quantifying similarity between motifs. Genome Biol 8: R24.

Harrow J, Frankish A, Gonzalez JM, Tapanari E, Diekhans M, Kokocinski F, Aken BL, Barrell D, Zadissa A, Searle S, et al. 2012. GENCODE: the reference human genome annotation for The ENCODE Project. Genome Res 22: $1760-1774$.

Hindorff LA, Sethupathy P, Junkins HA, Ramos EM, Mehta JP, Collins FS, Manolio TA. 2009. Potential etiologic and functional implications of genome-wide association loci for human diseases and traits. Proc Natl Acad Sci 106: 9362-9367.

Hu B, Lu Z, Li H, Chen Q. 2014. Convolutional neural network architectures for matching natural language sentences. In Advances in neural information processing systems 27 (NIPS 2014) (ed. Ghahramani Z, et al.), pp. 2042-2050, Montreal.

Ioffe S, Szegedy C. 2015. Batch normalization: accelerating deep network training by reducing internal covariate shift. J Mach Learn Res 37: 448-456.

Jin Y, Birlea SA, Fain PR, Ferrara TM, Ben S, Riccardi SL, Cole JB, Gowan K, Holland PJ, Bennett DC, et al. 2012. Genome-wide association analyses identify 13 new susceptibility loci for generalized vitiligo. Nat Genet 44: 676-680.

Kircher M, Witten DM, Jain P, O'Roak BJ, Cooper GM, Shendure J. 2014. A general framework for estimating the relative pathogenicity of human genetic variants. Nat Genet 46: 310-315.

Krizhevsky A, Sutskever I, Hinton GE. 2012. ImageNet classification with deep convolutional neural networks. In Advances in neural information processing systems 25 (NIPS 2012) (ed. Pereira F, et al.), pp. 1097-1105, Lake Tahoe, NV.

LeCun Y, Bottou L, Bengio Y, Haffner P. 1998. Gradient-based learning applied to document recognition. Proc IEEE 86: 2278-2324.

Lee D, Gorkin DU, Baker M, Strober BJ, Asoni AL, McCallion AS, Beer MA. 2015. A method to predict the impact of regulatory variants from DNA sequence. Nat Genet 47: 955-961.

Levo M, Segal E. 2014. In pursuit of design principles of regulatory sequences. Nat Rev Genet 15: 453-468.

Lupiáñez DG, Kraft K, Heinrich V, Krawitz P, Brancati F, Klopocki E, Horn D, Kayserili H, Opitz JM, Laxova R, et al. 2015. Disruptions of topological chromatin domains cause pathogenic rewiring of gene-enhancer interactions. Cell 161: 1012-1025.

Maurano MT, Humbert R, Rynes E, Thurman RE, Haugen E, Wang H, Reynolds AP, Sandstrom R, Qu H, Brody J, et al. 2012. Systematic localization of common disease-associated variation in regulatory DNA. Science 337: 1190-1195.

Melnikov A, Murugan A, Zhang X, Tesileanu T, Wang L, Rogov P, Feizi S, Gnirke A, Callan CG, Kinney JB, et al. 2012. Systematic dissection and optimization of inducible enhancers in human cells using a massively parallel reporter assay. Nat Biotechnol 30: 271-277.

Nair V, Hinton GE. 2010. Rectified linear units improve restricted Boltzmann machines. In International conference on machine learning, Haifa, Israel.

Okada Y, Wu D, Trynka G, Raj T, Terao C, Ikari K, Kochi Y, Ohmura K, Suzuki A, Yoshida S, et al. 2013. Genetics of rheumatoid arthritis contributes to biology and drug discovery. Nature 506: 376-381.

Patwardhan RP, Lee C, Litvin O, Young DL, Pe'er D, Shendure J. 2009. Highresolution analysis of DNA regulatory elements by synthetic saturation mutagenesis. Nat Biotechnol 27: 1173-1175.

Patwardhan RP, Hiatt JB, Witten DM, Kim MJ, Smith RP, May D, Lee C, Andrie JM, Lee S-I, Cooper GM, et al. 2012. Massively parallel functional dissection of mammalian enhancers in vivo. Nat Biotechnol 30: 265-270.

Pignon J-C, Grisanzio C, Geng Y, Song J, Shivdasani RA, Signoretti S. 2013. p63-expressing cells are the stem cells of developing prostate, bladder, and colorectal epithelia. Proc Natl Acad Sci 110: 8105-8110.
Pinello L, Xu J, Orkin SH, Yuan G-C. 2014. Analysis of chromatin-state plasticity identifies cell-type-specific regulators of H3K27me3 patterns. Proc Natl Acad Sci 111: E344-E353.

Pollard KS, Hubisz MJ, Rosenbloom KR, Siepel A. 2010. Detection of nonneutral substitution rates on mammalian phylogenies. Genome Res $\mathbf{2 0}$ $110-121$.

Ray S, Pollard JW. 2012. KLF15 negatively regulates estrogen-induced epithelial cell proliferation by inhibition of DNA replication licensing. Proc Natl Acad Sci 109: E1334-E1343.

Reid JE, Evans KJ, Dyer N, Wernisch L, Ott S. 2010. Variable structure motifs for transcription factor binding sites. BMC Genomics 11: 30 .

Ritchie GRS, Dunham I, Zeggini E, Flicek P. 2014. Functional annotation of noncoding sequence variants. Nat Methods 11: 294-296.

Roadmap Epigenomics Consortium, Kundaje A, Meuleman W, Ernst J, Bilenky M, Yen A, Heravi-Moussavi A, Kheradpour P, Zhang Z, Wang $\mathrm{J}$, et al. 2015. Integrative analysis of 111 reference human epigenomes. Nature 518: 317-330.

Rohs R, West SM, Sosinsky A, Liu P, Mann RS, Honig B. 2009. The role of DNA shape in protein-DNA recognition. Nature 461: 1248-1253.

Sawcer S, Hellenthal G, Pirinen M, Spencer CCA, Patsopoulos NA, Moutsianas L, Dilthey A, Su Z, Freeman C, Hunt SE, et al. 2011. Genetic risk and a primary role for cell-mediated immune mechanisms in multiple sclerosis. Nature 476: 214-219.

Segal E, Shapira M, Regev A, Pe'er D, Botstein D, Koller D, Friedman N. 2003. Module networks: identifying regulatory modules and their conditionspecific regulators from gene expression data. Nat Genet 34: 166-176.

Setty M, Leslie CS. 2015. SeqGL identifies context-dependent binding signals in genome-wide regulatory element maps. PLoS Comput Biol 11: e1004271.

Sherwood RI, Hashimoto T, O'Donnell CW, Lewis S, Barkal AA, van Hoff JP, Karun V, Jaakkola T, Gifford DK. 2014. Discovery of directional and nondirectional pioneer transcription factors by modeling DNase profile magnitude and shape. Nat Biotechnol 32: 171-178.

Slattery M, Zhou T, Yang L, Dantas Machado AC, Gordân R, Rohs R. 2014 Absence of a simple code: how transcription factors read the genome. Trends Biochem Sci 39: 381-399.

Snoek J, Larochelle H, Adams RP. 2012. Practical Bayesian optimization of machine learning algorithms. Adv Neural Inf Process Syst 2951-2959.

Stormo GD. 2000. DNA binding sites: representation and discovery. Bioinformatics 16: 16-23.

Szegedy C, Liu W, Jia Y, Sermanet P, Reed S, Anguelov D, Erhan D, Vanhoucke V, Rabinovich A. 2015. Going deeper with convolutions. arXiv:1409.4842 [cS.CV].

Thurman RE, Rynes E, Humbert R, Vierstra J, Maurano MT, Haugen E, Sheffield NC, Stergachis AB, Wang H, Vernot B, et al. 2012. The accessible chromatin landscape of the human genome. Nature 489: 75-82.

Tieleman T, Hinton G. 2012. Lecture 6.5-rmsprop: Divide the gradient by a running average of its recent magnitude. Neural Networks for Machine Learning. http://www.cs.toronto.edu/ tijmen/csc321/slides/lecture_ slides_lec6.pdf.

Voss TC, Hager GL. 2014. Dynamic regulation of transcriptional states by chromatin and transcription factors. Nat Rev Genet 15: 69-81.

Wang J, Zhuang J, Iyer S, Lin X, Whitfield TW, Greven MC, Pierce BG, Dong X, Kundaje A, Cheng Y, et al. 2012. Sequence features and chromatin structure around the genomic regions bound by 119 human transcription factors. Genome Res 22: 1798-1812.

Weirauch MT, Yang A, Albu M, Cote AG, Montenegro-Montero A, Drewe P, Najafabadi HS, Lambert SA, Mann I, Cook K, et al. 2014. Determination and inference of eukaryotic transcription factor sequence specificity. Cell 158: 1431-1443.

Welter D, MacArthur J, Morales J, Burdett T, Hall P, Junkins H, Klemm A, Flicek P, Manolio T, Hindorff L, et al. 2014. The NHGRI GWAS Catalog, a curated resource of SNP-trait associations. Nucleic Acids Res 42: D1001-D1006.

Whitaker JW, Chen Z, Wang W. 2015. Predicting the human epigenome from DNA motifs. Nat Methods 12: 265-272.

Wilanowski T, Caddy J, Ting SB, Hislop NR, Cerruti L, Auden A, Zhao L-L, Asquith S, Ellis S, Sinclair R, et al. 2008. Perturbed desmosomal cadherin expression in grainy head-like 1-null mice. EMBO J 27: 886-897.

Zhang X, Zhao J, LeCun Y. 2015. Character-level convolutional networks for text classification. In Advances in neural information processing systems 28 (NIPS 2015) (ed. Cortes C, et al.), Montreal.

Zhou J, Troyanskaya OG. 2015. Predicting effects of noncoding variants with deep learning-based sequence model. Nat Methods 12: 931-934.

Received October 4, 2015; accepted in revised form April 26, 2016. 


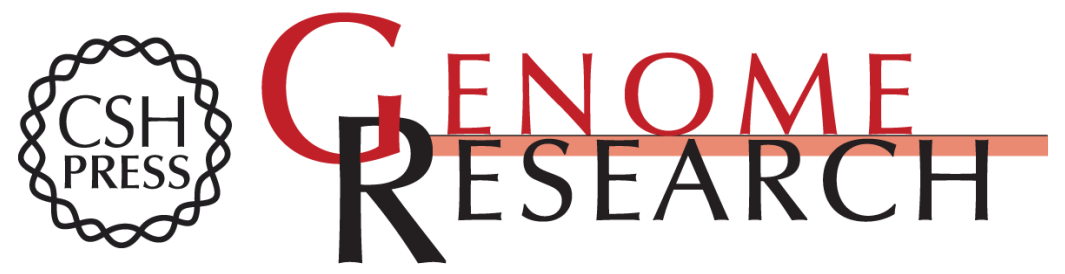

\section{Basset: learning the regulatory code of the accessible genome with deep convolutional neural networks}

David R. Kelley, Jasper Snoek and John L. Rinn

Genome Res. 2016 26: 990-999 originally published online May 3, 2016

Access the most recent version at doi:10.1101/gr.200535.115

Supplemental Material

References

Open Access

Creative Commons

License

Email Alerting Service
http://genome.cshlp.org/content/suppl/2016/06/10/gr.200535.115.DC1

This article cites 54 articles, 13 of which can be accessed free at: http://genome.cshlp.org/content/26/7/990.full.html\#ref-list-1

Freely available online through the Genome Research Open Access option.

This article, published in Genome Research, is available under a Creative Commons License (Attribution 4.0 International), as described at http://creativecommons.org/licenses/by/4.0/.

Receive free email alerts when new articles cite this article - sign up in the box at the top right corner of the article or click here.

\section{Affordable, Accurate Sequencing.}

To subscribe to Genome Research go to: https://genome.cshlp.org/subscriptions 